\section{Psoriasis: Kinder profitieren von Ustekinumab}

\author{
In mehreren Studien sind bei Erwachsenen mit Plaque-Psoriasis Sicherheit \\ und Wirksamkeit des Antikörpers Ustekinumab bestätigt worden. Jetzt \\ wurden die Studiendaten zur Prüfung des Präparates bei Kindern und \\ Jugendlichen veröffentlicht.
}

\begin{abstract}
n die CADMUS-Studie wurden 110 Kinder und Jugendliche mit mittelschwerer bis schwerer Plaque-Psoriasis - der PASI (Psoriasis Area and Severity Index) betrug mindestens 12, der PGA (Physician's Global Assessment) mindestens 3 - aufgenommen. Um an der Studie teilnehmen zu können, musste bei den Teilnehmern eine Phototherapie oder eine systemische Therapie indiziert sein oder eine topische Therapie versagt haben.

Die Patienten erhielten randomisiert zunächst zu Beginn und in Woche 4 sowie danach alle zwölf Wochen entweder ein Scheinpräparat $(n=37)$, die empfohlene Ustekinumab-Dosis $(n=36)$ oder die Hälfte der empfohlenen UstekinumabDosis $(\mathrm{n}=37)$, und zwar jeweils als subkutane Injektion. Als Standard wurde bei einem Körpergewicht von bis zu 60 kg
\end{abstract}

eine Dosis von $0,75 \mathrm{mg}$ pro kg Körpergewicht gewählt. Teilnehmer mit einem Gewicht zwischen 60 und $100 \mathrm{~kg}$ erhielten eine Dosis von $45 \mathrm{mg}$, mit einem Gewicht über $100 \mathrm{~kg}$ eine Dosis von $90 \mathrm{mg}$. In Woche 12 wurden die Patienten der Placebogruppe auf die Therapie mit dem Antikörper umgestellt. Primärer Endpunkt der Studie über ein Jahr war der Anteil der Patienten, bei denen in der Woche 12 ein PGA-Score von 0 (erscheinungsfrei) oder 1 (fast erscheinungsfrei) erreicht wurde. $\mathrm{Zu}$ den sekundären Endpunkten zählten PASI 75 und PASI 90.

Zwölf Wochen nach Studienbeginn lag mit der Standarddosis der Anteil der Patienten mit einem PGA von 0/1 bei 69,4\% in der Verumgruppe im Vergleich zu $5,4 \%$ in der Placebogruppe ( $\mathrm{p}<0,001)$. Den PASI 75 erreichten $80,6 \%$ und den
PASI 90 61,1\%, unter Placebo jedoch nur $10,8 \%$ und $5,4 \%$. Sowohl beim Parameter PGA als auch bei den PASI-Kriterien lagen die Ergebnisse bei halber Dosierung etwas niedriger. Der Anteil der Patienten, die über mindestens eine unerwünschte Wirkung der Therapie klagten, lag zu diesem Zeitpunkt mit dem Antikörper bei $44,4 \%$, in der Placebogruppe bei 56,8\%. Der stärkste Therapieeffekt wurde bis zur Woche 12 erzielt und hielt bis zum Ende des Follow-up nach einem Jahr an.

Fazit: Für Kinder und Jugendliche im Alter von 12 und 17 Jahren erwies sich der CADMUS-Studie zufolge zur optimalen Linderung der mittelschweren bis schweren Symptomatik eine Ustekinumabdosis von $0,75 \mathrm{mg}$ pro kg Körpergewicht als geeignet. Während der Studie traten keine anderen als die bereits bei Erwachsenen beobachteten unerwünschten Wirkungen auf. Peter Leiner

Landells I et al. Ustekinumab in adolescent patients age 12 to 17 years with moderate-tosevere plaque psoriasis: Results of the randomized phase 3 CADMUS study.

J Am Acad Dermatol 2015; 73: 594-603

\title{
Pachydermoperiostosis: wulstige Gesichtsfalten und Trommelschlegelfinger
}

Ein 32-jähriger Mann - genetisch ein Han-Chinese - hatte seit dem 15. Lebensjahr eine zunehmende Vergrößerung seiner Hände und Füße sowie eine Ausbildung wulstiger Gesichtsfalten an sich festgestellt (Abb. A). Er berichtete,
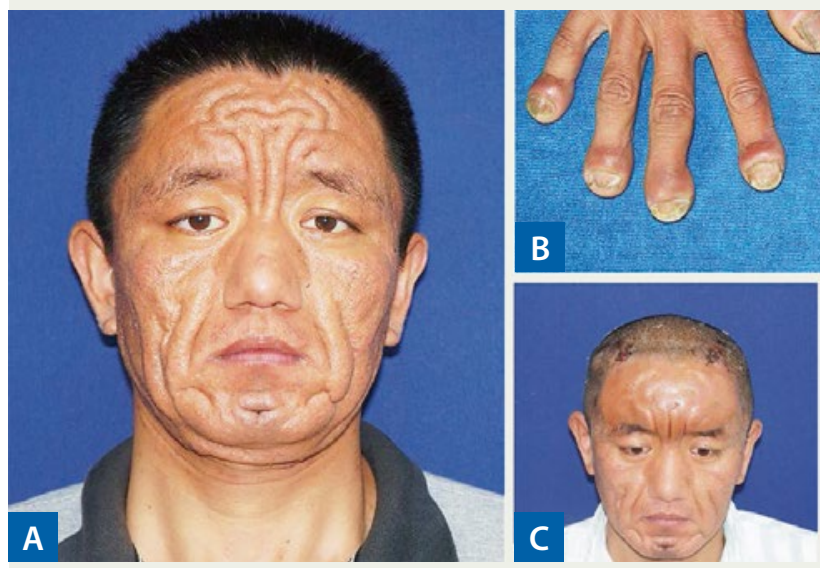

dass die Veränderungen bis zum 27. Lebensjahr fortgeschritten und seitdem zum Stillstand gekommen seien.

Die Gesichtshaut war verdickt, gefurcht und schien zu groß zu sein. Zudem hatte

A: Ausgeprägte Gesichtsfalten; B: vergrößerte Hände; C: unter der Stirnhaut implantiertes Expander-Kissen; D: Ergebnis nach Entfernung überschüssiger Haut. der Patient eine ausgeprägte Seborrhö. Im Bereich der Hände behinderten Weichteilschwellungen die Beweglichkeit. Insbesondere die Englieder der Finger und Zehen waren trommelschlegelartig aufgetrieben (Abb. B).

Man stellte die Diagnose einer Pachydermoperiostosis, einer seltenen genetischen Erkrankung, die mit Pachydermie, Trommelschlegelfingern und Periostitis assoziiert ist. Die Eltern des Patienten und ein Sohn hatten diese Veränderungen nicht. Eine genetische Testung wurde nicht durchgeführt. Die Therapie erfolgt in zwei Stufen. In der ersten Stufe wurde ein Silikonkissen unter die Stirnhaut implantiert, um die Haut zu spannen (Abb. C). Danach wurde die gefurchte und überschüssige Haut reseziert, sodass ein wesentlich besseres kosmetisches Ergebnis erzielt werden konnte (Abb. D). Prof. Hermann S. FüeßI

Liu C-Y \& Zhang Y-F. Pachydermoperiostosis. N Engl J Med 2014; 370: 1930 